\title{
Tendencia Suicida en Niños Accidentados
}

\author{
Renata Paula Morais dos Santos \\ Universidad de Ciencias Empresariales y Sociales, \\ B.A., Argentina.
}

\author{
Mônica Cristina Batista de Melo \\ Instituto de Medicina Integral Prof. Fernando Figueira, \\ PE, Brasil.
}

Resumen: La investigación intentó comprender los accidentes infantiles, considerando que esos eventos son, casi siempre, el resultado concreto de una denuncia de situaciones conflictivas y que pasan por hechos «ocasionales». Así, el objetivo fue investigar la posible tendencia suicida en niños entre los 6 y 12 años, hospitalizados en el Hospital da Restauração (Recife - PE/Brasil), por accidentes domésticos. Para el levantamiento de datos se utilizó un guion de entrevista semiestructurada, de carácter personal y familiar, destinado al niño y a su representante, como también la elaboración del Dibujo de la Familia, por parte del propio niño. Tras el análisis general de los resultados, puede afirmarse que, en los actos accidentales, aun cuando ocasionados por terceros, estaría reprimida una tendencia al suicidio. Los resultados indicaron una variedad de condicionantes personales y, principalmente, familiares, que pueden poner a los niños frente a un comportamiento de riesgo autodestructivo a corto y largo plazo.

Palabras clave: Accidentes, Ambiente Familiar, Factores de Riesgo, Niños, Suicidio.

\section{Tendência Suicida em Crianças Acidentadas}

Resumo: A investigação procurou entender o acidente com crianças, tendo em vista que esse evento é, quase sempre, o resultado concreto da denúncia de situações conflituosas e que passa por acontecimentos "ocasionais". Assim, o objetivo foi investigar a possível tendência suicida em crianças entre seis e 12 anos, hospitalizadas no Hospital da Restauração (Recife - PE/Brasil), por acidentes domésticos. Para a coleta dos dados, utilizou-se um roteiro de entrevista semiestruturada, de dimensões pessoais e familiares, destinada à criança e seu responsável, como também a realização, por parte da própria criança, do Desenho da Família. Após análise geral dos resultados, pode-se dizer que, no ato acidental, mesmo provocado por terceiros, estaria contida uma tendência ao suicídio. Os resultados indicaram diversos condicionantes pessoais e, principalmente, familiares, que podem colocar as crianças diante de comportamentos de riscos autodestrutivos a curto e longo prazo.

Palavras-chave: Acidente, Ambiente Familiar, Fatores de Risco, Crianças, Suicídio. 


\title{
Suicidality in Hilly Children
}

\begin{abstract}
The research tried to understand the accidents involving children considering that almost all are results of family conflicts, and comprehend if there's a desire of suicide in the risky act. This study aimed to investigate among children between 6 and 12 years old hospitalized due to domestic accidents if there is any possible tendency of suicide. Children hospitalized at the Hospital da Restauração in Pernambuco - Brazil made part of this study. A semi-structured interview was used to collect some information from children and their tutors as well a drawing made by the child showing the family environment. After analyzing all results it's possible to say that in the accident, even caused by others, there is a tendency to suicide. The results pointed out many personal and family sources that lead children to risky behavior and self-destructive acts in short or long-term.
\end{abstract}

Keywords: Accident, Family Environment, Risk Factors, Children, Suicide.

\section{Introducción}

Comprender el universo infantil y sus representaciones es cada vez más arriesgado. Los niños han avanzado mucho en sus capacidades complejas, dejando la comprensión del Ser mucho más difícil. Así, el interés por esos nuevos comportamientos de niños, en los que ellos distinguen perfectamente el juego de la realidad, hace con que cualquier persona se conmueva al embarcar en ese mundo tan distinto.

Esta investigación se propuso discutir un tema de carácter adulto que, infelizmente, ha alcanzado hasta a los propios niños: el suicidio. Por lo tanto, esta pesquisa aborda, con mucha cautela, y tomando en consideración el grupo que construye la adquisición de la personalidad infantil afirmando al individuo como sujeto: la familia, el motivo que está haciendo que los niños, antes considerados inocentes e inmaculados, estén involucrados en accidentes reales, en los que sus actitudes constantes están relacionados con la inminencia de la muerte, en un comportamiento aparentemente enmascarado por el ideal suicida, tema casi intocado y disimulado por la negación adulta.

La familia muchas veces es mencionada por Winnicott (2011) como representante de un grupo cuya composición se relaciona directamente con la estructura de la personalidad del individuo, siendo la primera agrupación responsable por el crecimiento individual. La familia participa del lugar donde los niños descubren sentimientos de amor y odio, y donde ellos pueden esperar simpatía y tolerancia, así como la exasperación que ocasionan. Además, muchas cosas han sido descubiertas y transmitidas a esos niños en ese ambiente; a fin de cuentas, nunca se ha hablado tanto de violencia contra los niños como actualmente.

La violencia puede ocurrir en un hogar con problemas, como los que vive una persona que sufre de una enfermedad crónica (por bebida, enfermedad somática y mental), o como los problemas de relacionamiento entre familia y la sociedad. Tal violencia, aunque inexplicable para el niño, es la que, muchas veces, lo lleva a sentirse merecedor de castigos, o buscar medios que le permitan librarse de ese ambiente amedrentador, usando, casi siempre, comportamientos autodestructivos como los accidentes y el propio suicidio, en el intento de liberarse y dar fin a las situaciones conflictivas de la vida.

En el universo que rodea a los niños muchas veces se observa que hacen verdaderos "malabarismos" para ser, de alguna manera, vistos y oídos. El accidente puede, sin duda alguna, ser un intento de llamar la atención y, con eso, denunciar o librarse de algo que los maltrata. Por eso, el adulto debe ausentarse un poco de su propio mundo (en el cual muchas veces "ingenuamente" ignora los comportamientos infantiles, por creer que los niños son tan dulces e inocentes que no tienen problemas o dificultades suficientes para atentar contra la propia vida) y observar lo que se manifiesta tras los gritos silenciosos de esos pequeños que exigen atención y alivio. 
Así, este trabajo fue elaborado con el objetivo de descubrir posibles tendencias suicidas en niños, de 6 a 12 años, hospitalizados por accidente doméstico, teniendo como base el contexto familiar y la interrogante sobre los motivos que llevan a que el niño se envuelva en una situación de peligro. ¿Cuál será la verdadera intención de los niños? ¿Serán las ganas de morir propiamente dichas, o un deseo de despertarse en un mejor ambiente, con nuevas posibilidades? ¿Sería un comportamiento consciente, o inconsciente con la alternativa de librarse de algo sofocante? ¿Sería la pérdida del mito de la inocencia?

Este estudio pretendió, a lo largo de las secciones, proporcionar una respuesta a las numerosas preguntas acerca de los accidentes de menores, además de añadir conocimientos a la gran laguna existente en la literatura actual con relación al tema, tornando posible el acto lesivo como un probable intento enmascarado de suicidio y, con eso, hacer con que se piense sobre crear medios que puedan prevenir que el mismo se consume. De esta manera, la investigación abordó conceptos sobre la familia, el desarrollo infantil, el suicidio, la compresión de los accidentes domésticos en la infancia y sus explicaciones psicológicas.

\section{Antecedentes y Planteamiento Teórico}

Poco se habla sobre el tema de suicido de niños. Lo que sí se sabe es que la edad en que se viene cometiendo tal acto autodestructivo está disminuyendo cada vez más. La creencia de que los menores puedan involucrarse en estos comportamientos parece aún subestimada, principalmente porque los conceptos de muerte están más allá del alcance verbal de un niño muy pequeño - esto, sin embargo, no significa necesariamente que no tenga comprensión acerca del tema.

Existen estudios sobre el desarrollo de la percepción de la muerte en niños pequeños, de entre 3 y 5 años de edad, de los que no se esperaría conocimiento alguno al respecto, una vez que esta idea parecería muy abstracta, lejana a su vivencia y conocimiento. Paradójicamente, autores como Fensterseifer y Werlang (2003) sustentan que en todas las edades hay percepciones diferentes con respecto a la muerte. La idea parte de los juegos infantiles, en los cuales los niños desde los 3 meses de edad son "sensibles a la idea de ausencia/vacío; y la presencia se constituye como la capacidad de establecer la distinción de estar ahí y no estar" (p. 42).

Torres (1999), en su investigación sobre el desarrollo del concepto de muerte en los niños, muestra que tanto la edad cronológica como la mental se relacionan significativamente en el desarrollo del concepto. Es cierto que el niño, en las diferentes etapas de su desarrollo, es capaz de comprender acerca del tema. Mientras la definición de la muerte continúa siendo una tarea muy difícil, tanto para adultos como para niños, y mientras que hay una negativa que rodea el tema, el número de muertes, sobre todo, por suicidio, ha aumentado crónicamente en los últimos años. De ahí la importancia de comenzar a romper con el tabú y hablar más sobre el tema.

\section{El Suicidio Infantil y los Accidentes}

Etimológicamente la palabra suicidio proviene del latín suicidium, que se divide en sui (sí mismo) y caedes (acción de matar), del verbo caedo, is cedici, caesum, caedere. Las investigaciones dicen que el origen estuvo en Inglaterra, siendo atribuido a Sir Thomas Browne, que publicó el término en su libro Religio Medici en 1643. Por otro lado, algunos ingleses le atribuyeron la paternidad a Charleton (1651) y otros a Edward Phillips (1662), en el diccionario filosófico New Words of the World. Se cree que en portugués la palabra fue usada desde 1836 según el Diccionario Houaiss (Corrêa, \& Perez Barrero, 2006, p. 3).

De cualquier forma, Durkheim (2009) fue nombrado como el sociólogo precursor de los estudios sobre el suicidio y dictó clásicamente la definición de este acto como "todo caso de muerte que resulte, directa o indirectamente, de un acto, positivo o negativo, realizado por la victima misma, sabiendo ella que debería producir este resultado" (p. 10).

De forma general, la palabra suicidio es usualmente usada para definir el acto-acción de poner, por voluntad propia, fin a la propia vida, por medio casi siempre de comportamientos autodestructivos revelando el deseo de morir; es un acto de renuncia a la vida, una acción que no podría ser más personal para un individuo (Stengel, 1987). Por otro lado, autores como Kreitman (1988, citado por Marchiori, 2006) argumentan que no existe una definición clara para el suicidio, ya que la motivación, que es el tema principal del acto, no es bien definida. Por esto, este autor deja un espacio en blanco sobre los motivos 
conflictivos que dan significado a dicho comportamiento. No obstante, es innegable que el suicidio es una acción que se relaciona directamente con otras personas en su forma más agresiva. Un acto propiamente del ser humano que se consagra, casi siempre, con conocimiento de causa (p. 3).

Según la Organización Mundial de Salud, se estima que en 20201.53 millones de personas en todo el mundo morirán por acto suicida, lo que significa un suicidio cada 20 segundos; por otro lado, cada 1 o 2 segundos alguien intentará cometer suicidio (Bertolote, \& Fleischmann, 2002). Teniendo en cuenta que hay gran falla en los registros sobre los hechos suicidas, los números indicados poden ser mayores a los estimados. Además, muchos casos son, todavía, considerados como accidentes o son registrados insensiblemente de forma errada, principalmente cuando se refieren a infantes, pues ellos están rodeados siempre del tabú sobre la capacidad autodestructiva de los menores.

En Brasil, las informaciones sobre mortalidad por suicidio son registradas en la sesión de Causas Externas da Clasificación Internacional de Enfermedades de la OMS, lo que dificulta la veracidad de los números divulgados en el país, una vez que las muertes por suicidio se mezclan con otras muertes no decurrentes de enfermedades biológicas, incluyendo, además de los suicidios, las muertes por violencia y accidentes (Meleiro, Teng, \& Wang, 2004).

Frente a las implicaciones clínicas involucrando niños y al gran vacío existente en la literatura acerca del tema, se hace necesario cuestionar si serán realmente actos accidentales, ya que es alarmante el gran aumento de la mortalidad atribuida a dichos "accidentes" involucrando infantes.

Datos de ONGs, como la ONG Criança Segura en Brasil, sostienen que, aproximadamente, 5 mil niños menores de 14 años mueren por accidentes o lesiones no intencionadas todos los años (ONG Criança Segura, 2011). Una proporción desconocida de actos suicidas queda en este registro como accidente, por lo que hay una preocupación por los actos accidentales infantiles y por el fallido registro referente a las verdaderas causas de muertes en menores (Cassorla, 1991).

Así, dependiendo de las circunstancias, un suicidio puede pasar fácilmente desapercibido o ser entendido como un acontecimiento normal. Por este motivo, Fensterseifer y Werlang (2003) llaman la atención para los accidentes involucrando la muerte de niños y resaltan que los pequeños pueden estar involucrándose en situaciones de riesgo, incluyendo en ese contexto el indiscutido e intocado suicidio infantil.

Cualquier conducta arriesgada realizada por niños que ya tengan discernimiento de los peligros debe ser considerada, principalmente porque el suicidio consumado es frecuentemente antecedido por un intento. Así, teniendo los conceptos de muerte ya definidos, y la consolidación de estos de acuerdo con la edad y las experiencias del niño, se puede percibir que los infantes, desde las edades más precoces, tienen la capacidad suficiente para ejecutar su deseo de poner fin a la propia vida.

El suicidio en los niños se transforma en una de las maneras más trágicas de liberarse de situaciones que muchas veces son conflictivas y que se relacionan con exigencias adultas. Es cierto, "no existe total acuerdo sobre las características propias del suicidio infantil, si bien se admite generalmente que las conductas suicidas durante esta época de la vida están muy determinadas por la vivencia del momento presente" (Polaino-Lorente, \& Heras, 1990, p. 224)

Los factores de riesgo suicidas son comúnmente estudiados a través de datos epidemiológicos. Rescatando informaciones vinculadas a los casos de suicidios que se consumaron, por métodos como las Autopsias Psicológicas, es posible comprobar factores que se conectan con estados psicológicos, psiquiátricos, cuadros clínicos, socioeconómicos e, inclusive, características sobre el ambiente familiar de los involucrados. Es así que autores como Dias (1997) relacionan el acto suicida directamente con la unidad familiar y el medio social próximo al individuo.

La muerte auto-provocada sigue abriendo cantidad de interrogantes directamente relacionados a los actos suicidas, una vez que la muerte lleva consigo las respuestas y deja los interrogantes sobre sus motivos a través de información que la víctima dejó para terceros. Por esto, la persona que realizó el acto no es la única a ayudar en la interpretación de su conducta. Los que sobreviven, y su ambiente, se transforman en una fuente de investigación en la búsqueda de entender los motivos para tal comportamiento.

Para el psicoanálisis, toda actividad humana es constituida por factores vitales individuales que van al encuentro de una determinada realidad externa. Frente a esto, se debe reconocer que no solamente existen factores familiares y sociales, poseedores de 
una fuerza suficiente para interferir e influenciar en un comportamiento accidental humano, en los acontecimientos accidentales, sino también influencias provenientes del inconsciente; así, los accidentes, por lo tanto, serían consecuencias de un conjunto de factores y no solamente de una casualidad.

Los accidentes pueden ser vistos como una acción corporal que está dando una respuesta a ese mundo interno conflictivo. La causa de los accidentes generalmente se atribuye a factores puramente externos, y muchas veces así es. Pero el psicoanálisis abre puertas y propone diferentes significados para el accidente en la medida en que se presenta como un proceso que lleva en sí un origen, un desarrollo, una finalidad y un sentido.

Algunos autores, de hecho, amparan la idea de que muchos accidentes no son simple casualidad, ya que creen que sirven como proceso para el inconsciente. Varios mecanismos provenientes de este inconsciente se colocan en un funcionamiento materializado en sentimientos reprimidos, muchas veces llenos de culpa y acusaciones, presentando un cierto grado de necesidad para el autocastigo, o deseos de venganza, valorización o protección, convirtiéndose en fuente de escape en los accidentes (Amaral, \& Mattioli, 2003).

Además, los accidentes se puedan mostrar como explicación de una fuerza interna que busca escapar de un desequilibrio a veces desconocido y de gran intensidad. Los sujetos que buscan en los accidentes la forma de organizarse internamente (y claro, inconsistentemente), generalmente han vivido situaciones que requieren cambios en la forma de vivir. Así, todo cambio de importancia vital para una persona la invita a transitar por un estado de turbulencia y, generalmente, de agitación (Granel, 2009).

De esta manera, se muestra que lo imprevisto, en los acontecimientos azarosos de los accidentes, no es tan imprevisible; se ve que es a través de este supuesto "azar" que el sujeto parece encontrar la salida de los conflictos vividos, padeciendo y provocando los accidentes como una forma de defensa, muchas veces, viendo como deficiencias los actos ocurridos en los procesos inconscientes y conteniendo un sentido mucho más allá de lo ocasional. Entonces, saliendo del plano aparente presente en los actos accidentales, se podría decir que son estos, acontecimientos de representaciones sólidas. Representaciones motivadas por posibilidades entre las cuales el inconsciente se hace presente.
¿Entonces sería el accidente una vía de escape, tal como lo es el suicidio, que se presenta como una salida también a los conflictos psíquicos? ¿ No estaría el sujeto, por pequeño que este fuese, viviendo un fuerte conflicto inconsciente que lo haría capaz de, "accidentalmente", herirse a sí mismo a través de sus acciones equivocadas?

Parece que no habría error al pensar de tal forma y, tampoco, al conectar los actos accidentales a los actos suicidas, una vez que los propósitos inconscientes de auto-aniquilación esperan siempre una ocasión oportuna, para evitar la acción de fuerzas defensivas que podrían llevar a la persona a desistir de su propósito suicida.

\section{Familia y el Desarrollo Infantil}

Cuando se habla de familia, se visualiza un modelo familiar en el cual incluimos de inmediato las figuras paternas y los hijos, formándose así el conocido núcleo familiar. El modelo familiar se viene transformando a través de los tiempos, adaptándose a los cambios -en la medida en que sufre las influencias de nuevos acontecimientos-, generando así, un concepto modificado que se adapta según el tipo de sociedad, tiempo y estructura social (Pinheiro, \& Biasoli-Alves, 2008).

Familia, por otro lado, engloba un término amplio y complejo. Sin duda, ella es una institución social primaria de gran influencia que sufre modificaciones a lo largo del tiempo. Los lazos de sangre y parentesco siempre fueron parámetros que definían con precisión la configuración familiar; sin embargo, con el pasar del tiempo, creció el nivel de complejidad en la definición de estas configuraciones (Wagner, \& Levandowski, 2008).

Pensar en definir familia se transforma en una tarea muy difícil, ya que se corre el riesgo de excluir algunas formas diferenciadas e inéditas de organizaciones familiares. Actualmente no se puede imaginar una familia estructurada que esté formada exclusivamente por un grupo de personas ligadas por descendencia, por algún parentesco o por múltiples lazos relacionados con el mantenimiento de sus características durante generaciones, ya que la configuración familiar nuclear viene siendo fuertemente disuelta por los cambios de la modernidad.

Las nuevas formas de construcción de familia se muestran cada vez más ampliadas, y esas relaciones 
familiares que se generan ejercen claramente cambios sobre sus miembros y sobre la sociedad. Una cosa es cierta, la familia, independientemente de los nuevos modelos que vengan, aún será familia (Pinheiro, \& Biasoli-Alves, 2008).

Organizada sobre la base de las relaciones interpersonales, la familia se incluye entre las instituciones sociales básicas para el desarrollo. Cada familia tiene la misión de dar su propio significado a su espacio y escribir su historia. Desafortunadamente, muchas veces estas relaciones pueden provocar sufrimiento, ya que no podemos olvidar que la familia es un gran escenario en el cual se viven intensamente emociones y experiencias que marcan. Es el lugar donde se puede convivir con amor y odio, con alegría y tristeza, donde se vivencia el desespero y se aguarda la esperanza. La familia es la única institución capaz de proporcionar (o no) equilibrio entre sus miembros (Wagner, 2002).

Es por esto que Winnicott (2011) ya mencionaba que la unidad familiar posee un crecimiento y desarrollo particular. Cada miembro cambia y crece emocionalmente, aunque el crecimiento físico ya se haya establecido. Es así que en la familia el sujeto se construye, ya que el crecimiento y el desarrollo de cada familia van siendo traspasados a las nuevas generaciones; cada miembro heredará a otro, a cada momento, un poco de sí, un poco de su construcción. La familia es el primer ambiente en el cual se construye y desarrolla la personalidad de cada ser humano. Hay que destacar que hablar sobre la familia es depararse con un grupo de gran fuerza y valor, participante activo y coadyuvante en el desarrollo infantil, sobre todo en la formación y construcción de la personalidad de las nuevas generaciones, específicamente de los niños.

Es prácticamente imposible pensar en el desarrollo infantil lejos del contexto familiar, pues es el grupo esencial en la formación de estos nuevos individuos y su comportamiento individual. La familia tiene que ser vista como el más influyente conjunto de sistemas sobre el desarrollo de cualquier niño, siendo también uno de los más poderosos aparatos de socialización para el desarrollo saludable de un sujeto. Teóricamente, lo más importante en el desarrollo de un niño es un ambiente seguro y saludable; un ambiente capaz de ofrecerle subsidios básicos para su crecimiento y para la maduración de su ser.

Por esto, ¿cómo establecer un prototipo único de modelo familiar que pueda ser considerado como un ejemplo de familia saludable y suficientemente buena, para cubrir las necesidades básicas de los niños frente al aumento de variantes en los modelos familiares y reestructuraciones existentes? Lo que parece ser más coherente frente a esto es que es importante buscar en todos aquellos que pretendan contribuir al desarrollo de los niños que tengan, por lo menos, recursos emocionales, sociales y materiales adecuados para este fin (Marin, \& Piccinini, 2009).

Es cierto que un mismo ambiente puede provocar en los mismos niños efectos diferentes, ya que debemos recordar que cada uno posee diferentes características innatas. Vale observar que ambientes similares pueden estar conectados a resultados diferentes. Así, la capacidad y salud de cada individuo en el ambiente familiar también depende de las características individuales y, específicamente, de factores como ejecución de papeles particulares y delimitación de la función de autoridad de las figuras significativas, los cuales son esenciales para un funcionamiento familiar saludable y para el bienestar de sus miembros (Oliveira, Siqueira, Dell'Aglio, \& Lopes, 2008).

Winnicott (2011) diría que gran parte de los factores que generan complicaciones en la vida familiar proviene de las actitudes que los padres toman cuando se agota su capacidad de sacrificar todo por los hijos. Por otro lado, negarles a los niños el acceso a sus derechos no puede ser justificado por esa actitud. Al final, la familia tiene que ser responsable del desarrollo y maduración biopsicosocial de sus individuos.

De cualquier forma, al contrario de lo que se piensa, varias veces los familiares se convierten en obstáculos en la vida y en el desarrollo de los hijos, y no es extraño que estas pequeñas cosas se generen bajo el peso de relaciones en los ambientes domésticos que pueden llevarlos hasta la muerte (Winnicott, 2011). Muchos niños, desafortunadamente, son expuestos a situaciones de vulnerabilidad y riesgo que atacan el desarrollo, dejándolos susceptibles a una infancia rodeada por eventos comprometedores y cargados de sufrimiento y de daños para su desarrollo.

Existen los que creen que algún día existió una familia tan estable y buena que solamente ofrecía amparo, seguridad y buenos patrones morales para los niños y sus miembros. Desafortunadamente, hoy en día, ya se puede ver que esta familia está cambiando frente a las constantes observaciones de 
sus productos: sujetos, principalmente niños, angustiados, depresivos, delincuentes y antisociales.

La familia actual parece que no está asumiendo su papel de promover la salud y el bienestar a sus integrantes, que involucraría el desarrollo de actividades de protección, seguridad y cuidados. Es común relacionar las vivencias de los niños a los cambios en el ambiente familiar. Separaciones, divorcios, nuevos matrimonios o familias monoparentales son, de hecho, eventos cargados de sufrimiento que pueden desencadenar innumerables perjuicios, por encontrarse en un periodo de cambios en el ciclo de la vida de la familia que involucra directamente a los miembros involucrados, afectando su desarrollo.

Estas situaciones, sin duda, son un ejemplo de eventos estresantes que pueden alterar significativamente el ambiente, pudiendo ocasionar un nivel alto de tensión influenciando el comportamiento y las respuestas de vida de los individuos. Estos son algunos, entre muchos, factores de riesgo que colocan en prueba el desarrollo de los niños, ya que, como eventos estresantes, están asociados a la espera de resultados negativos o indeseables.

Diversos autores entienden como factores de riesgo las condiciones o variables que pueden comprometer la salud, bienestar o performance social del sujeto asociado a la alta posibilidad de que ocurran resultados indeseables y negativos para el desarrollo. También pueden ser definidos como toda clase de eventos que aumentan la probabilidad de que el individuo presente problemas físicos, sociales, de comportamiento o emocionales. Tales factores pueden incluir tanto atributos biológicos y genéticos del niño o de la familia, como factores presentes en el ambiente económico, psicológico y familiar que tienen una gran posibilidad de ocasionar daños significativos en el desarrollo de los niños (Delvan, Becker, \& Braun, 2010).

Es importante resaltar que los factores de riesgo también son reconocidos como procesos, variables individuales, ambientales o contextuales que aumentan la vulnerabilidad del niño para resultados negativos que dependen de la cantidad de factores presentes, así como del tiempo de exposición a estos eventos, sin ser un solo factor el único relevante por más grave que sea (Ramires, Passarini, Flores, \& Santos, 2009).

Ligado a eso, la literatura nos sorprende con la alta incidencia de accidentes domésticos infantiles que, casi siempre, aparecen asociados a violencias domésticas sufridas, indicando así, que es en estos ambientes en los que los niños están siendo víctimas de descuidos, privaciones y agresiones que ocurren, sobre todo, bajo la cultura del silencio y de la tolerancia social.

El ambiente familiar debería ser un ambiente que proporciona ventajas para la salud y el bienestar de los niños en todos sus aspectos. Empero, algunas características de este lugar, como el estilo de vida, factores educacionales, económicos, sociales y culturales, pueden estar manteniendo estrecha relación con los accidentes domésticos (Nascimento, Sousa, \& Chacon, 2012).

Igualmente, autores defienden que los accidentes guardan relación con una amplia red de factores que van más allá del ambiente, tales como las condiciones físicas, culturales y sociales de la familia: el estilo de vida, condiciones de vida y de trabajo de los padres, urbanización, marginalidad, desempleo, desigualdad social, superpoblación, miseria, educación, estrés, malas condiciones de vida, vigilancia insuficiente, entre otros (Martins, 2006). Otros factores de riesgo también pueden estar conectados a la características personales de los niños: temperamento, personalidad (hiperactividad, agresividad, impulsividad, distracción) y capacidades psíquicas y físicas (Nascimento et al., 2012).

Frente a todo esto, son cuestionables las motivaciones que estarían llevando a los niños a actos constantes para lesionarse en una edad donde ya hay comprensión de los riesgos. ¿Serán los agentes externos, funcionando como torturadores, los que abren las puertas a fantasías infantiles que, subyacentes al acto accidental, corren en dirección a otra vida, a un nuevo mundo lleno de nuevas posibilidades, al retorno al seno materno o inclusive, al deseo de punición, de venganza, de proporcionar culpa y causar sufrimiento a los otros y a la sociedad? (Cassorla, 2005).

Quizás el accidentarse sea una tentativa de llamar la atención y, con eso, denunciar o librarse de algo sofocante del mundo, estando ahí, implícito, de alguna forma, el deseo de muerte o de lastimarse como una fuga y denuncia de este ambiente. De cualquier manera, se cree que en el acto de lastimarse el sujeto no desea exactamente su muerte, razón por la cual los actos de autolesión se producen en ambientes domésticos donde es posible tener socorro. 


\section{Método}

Esta es una investigación exploratoria de tipo cualitativo y transversal, con base en procedimientos de registro, análisis y correlación de datos. La investigación cualitativa ofrece recursos para crear datos descriptivos y permite la observación del comportamiento de los sujetos, objeto de la investigación, por lo cual se puede describir profundamente la realidad, teniendo en cuenta lo que expresan estos sujetos.

Esta investigación se realizó en el Hospital da Restauração (HR), localizado en la ciudad de Recife, estado de Pernambuco (Brasil), y considerado la mayor unidad de la red de salud pública estatal. Los sujetos participantes fueron niños de 6 a 12 años de edad, que se encontraban hospitalizados debido a accidentes domésticos y que presentaban capacidad de hacer un dibujo.

Antes de todo, el proyecto de la investigación y los instrumentos utilizados fueron analizados y aprobados, por el Comité de Ética en Investigación del Centro de Estudios del propio Hospital, permitiendo iniciar la investigación. Previamente al procedimiento de la colecta de datos, los participantes fueron debidamente informados sobre la naturaleza y las características de la investigación. Todas las informaciones estaban presentes en un Término de Consentimiento Libre y Esclarecido leído y explicado antes de la firma del responsable.

La recolección de datos se realizó con la utilización de dos guiones de entrevista semiestructurada y un dibujo de la familia. En un primer momento, hubo una entrevista semiestructurada con el familiar responsable por el niño. En seguida, se solicitó a los niños dibujar "la familia”, con el objetivo de establecer un rapport y observar la dinámica y problemática del niño en su ambiente familiar. Después se realizó con los mismos niños una entrevista semiestructurada, de dimensión personal, en el intento de justificar y valorar su comprensión sobre el suicidio y los comportamientos conectados a los accidentes.

Las entrevistas fueron grabadas y transcritas. Los registros fueron guardados adecuadamente para mantener la confidencialidad de las informaciones. Para el análisis del material recolectado, fue realizada, inicialmente, una lectura general de los documentos -según lo propuesto por Franco (2005)-; en seguida, estos datos fueron analizados y discutidos para alcanzar los objetivos propuestos, siempre con referencia a la vasta bibliografía utilizada.

\section{Resultados y Discusión}

Participaron de la investigación diez niños que estaban internados en el Hospital da Restauração por accidentes domésticos. Eran participantes de ambos sexos, con edades entre los 6 y 12 años, escolaridad entre la pre-escuela y el $5^{\circ}$ año básico, residiendo con sus progenitores y otras figuras como hermanos, abuelos y tías.

Para la participación en la investigación, se aceptó que tanto los padres, como los familiares próximos y tíos, que estaban presentes en el hospital con los niños, respondieran al cuestionario sobre la familia. Lo anterior, porque el cuestionario fue, también, basado en el método de la Autopsia Psicológica (en el que se puede realizar la investigación con terceros sin la presencia del sujeto-víctima).

Los resultados encontrados indicaron condicionantes personales $\mathrm{y}$, principalmente, familiares, que podrían colocar fácilmente a estos niños ante comportamientos de riesgo autodestructivos a corto y a largo plazo. Las mismas características encontradas en el grupo estudiado, estuvieron presentes en otros estudios realizados en poblaciones que habían intentado, o realizado, actos suicidas.

Como eventos estresantes, estuvieron presentes en el grupo características como: padres separados, número significante de hijos, problemas psiquiátricos en la familia, y presencia de hijos no deseados. Diversos autores, como Basile (2005), Delvan et al. (2010), Oliveira et al. (2008), García, Muro, Prado, Martinez, \& Calvo (2007), confirman estos factores como preocupantes, tanto para el desarrollo de un niño, como para los futuros riesgos suicidas. En el embarazo no deseado, por ejemplo, puede haber, de forma explícita o discreta, diferentes tipos de manifestaciones de rechazo del niño, producto de las actitudes de sus padres, de tal manera que él no se siente bienvenido (Barrero, Nicolato, \& Corrêa, 2011).

Incluyéndose en los eventos de vida estresantes encontrados, estuvieron presentes en el grupo y en otros estudios propuestos por Fensterseifer y Werlang (2003) y Ramires et al. (2009): el bajo nivel escolar de los padres, el abuso de drogas (entendiendo estas como lícitas e ilícitas), el abandono y el distanciamiento de la figura paterna. Pequeños puntos que generan un ambiente de estrés desencadenado graves problemas que comprometen el ambiente familiar y el desarrollo humano (Delvan et al., 2010). 
Otro punto que llama la atención sobre los riesgos propios al niño y sus padres, es el hecho de que los genitores sean adolescentes en el momento de concepción del hijo, ya que se entiende que la escasa edad en los progenitores, relacionada a inmadurez biológica y psicológica, influye de manera importante en la educación de los hijos (Barrero et al., 2011).

No obstante, no muchas madres de los niños participantes tuvieron su embarazo en la adolescencia. Pocas tuvieron entre 17 y 18 años; por su parte, todos los padres fueron mayores de 23 años. Unglert, Siqueira y Carvalho (1987), presentan en su investigación que las familias con la presencia de accidentes traían padres y madres mayores. O sea, se puede entender que para el riesgo accidental de suicidio, una baja edad de los padres influye tanto como una edad más avanzada. Esto refuerza que las figuras de los padres configuran el principal núcleo social del niño, teniendo gran influencia en el proceso de desarrollo cognitivo, social y psicológico de los menores, independientemente de su edad en el momento de la concepción (Pinheiro, \& Biasoli-Alves, 2008, p. 30).

Así como la gran cantidad en el número de hijos desvía la atención y el cuidado necesario para cada uno de ellos, también la posición ordinal del niño en la familia parece proporcionar un cierto riesgo suicida para estos sujetos. Algunos niños participantes de la investigación se encontraban en la posición de primogénitos o menores y, de hecho, aún existen pocos estudios sobre el tema. No obstante, Resmini (2004), en la búsqueda de comportamientos suicidas en la adolescencia, encontró el predominio de este comportamiento en los primogénitos, seguidos por los menores e hijos únicos.

Así, parece que la posición en el orden de nacimiento siempre debe ser considerada, principalmente en relación al sexo del individuo, de sus hermanos y la distancia que separa a un nacimiento del otro. Al final, el que detiene inicialmente toda la atención colocada en sí, con la llegada de los hermanos, la divide, y esto puede ser vivenciado como una pérdida o abandono, dificultando los procesos de separación-individualización (Resmini, 2004).

Sobre separación, el grupo reveló que estaba presente la experiencia con relación al tema de la muerte. Los niños relataron haber vivido la muerte de, por lo menos, un familiar próximo, estando entre estos: madres, abuelos, tíos, hermanos, amigos entre otros, que eran parte de las relaciones afectivas del niño.
Entre los tipos de muertes relatadas, solamente no hubo presencia de muerte por hechos accidentales. Muertes violentas, como los homicidios y suicidios estuvieron presentes y, en algunas familias, aparecieron juntos. Una preocupación a adicionar es que la historia de suicidio en la familia es marcada como un gran factor de riesgo tanto en jóvenes como en adultos (Basile, 2005; Barrero et al., 2011).

Hubo relatos de que las informaciones entregadas a los niños sobre este tema cruzaron las líneas directas e indirectas de las mismas, dejando abierto el camino para fantasías que, fácilmente, pueden: llegar a generar miedos como ir a dormir, por la semejanza de la muerte con el sueño; o inclusive generar un acto suicida en el intento de reunirse e ir al encuentro del ser amado. Según Adorno (1994), los niños mostraron cierta curiosidad en saber para dónde se va cuando se muere, y la respuesta debe ser objetiva en relación al destino del cuerpo y no al del alma, considerando que es el cuerpo lo que el niño conoce.

Así, la presencia de muerte en el contexto familiar es un factor importante para riesgos suicidas, principalmente, si esta muerte es de un familiar muy cercano, pues aumenta la predisposición suicida en un individuo por los próximos cuatro a cinco años, especialmente, si cuenta con poco soporte familiar (Meleiro et al., 2004).

Igual, algunos niños reportaran a una muerte física 'inexistente', fantaseada frente a la separación de los padres; en cuanto a esa pérdida, resultante de la separación de los padres, la literatura abordada es unánime en mostrar que ese acontecimiento se convierte, tanto en la infancia como en las consecuentes fases de la vida, en un punto de importancia para los intentos de autoeliminación de un sujeto. Estudios como los de Bella, Fernández y Willington (2010) muestran que en el grupo con intento de suicidio la estructura familiar que prevalecía era la monoparental materna. Esto significa que no tener las figuras paternas como figura de autoridad genera 15 veces más riesgo de suicidio.

Además, en los participantes, en su grupo etario, no hubo ninguna prevalencia entre categoría de sexo, distribuyéndose igualmente entre aquellos del sexo femenino y del sexo masculino. Sin embargo, la literatura destaca prevalencia en los acontecimientos accidentales, y en los actos suicidas consumados, marcadamente, en la población masculina. La población femenina se destaca, mucho más, por los 
intentos suicidas que ocurren tres veces más que en la masculina. Según Amorim et al. (2012), el predominio de estos acontecimientos en la categoría masculina puede producirse debido a diferencias de las actividades realizadas. Los niños se involucran en actividades y juegos más dinámicos y de mayor contacto físico; las niñas, por su parte, parecen relacionarse más con actividades más tranquilas.

Como no podría ser diferente, todos los incidentes ocurrieron alrededor de sus residencias, en distintos ambientes, principalmente en la cocina, seguida por el patio de la casa; las quemaduras fueron los eventos más predominantes. De hecho, gran parte de las quemaduras ocurrió en la cocina, que es reconocida por diversos autores como un local de gran riesgo para la población infantil (Paes, \& Gaspar, 2005; Vendrusculo, Balieiro, Echevarría-Guanilo, Farina Junior, \& Rossi, 2010).

Aunque las quemaduras no se encuentran entre los accidentes infantiles o medios de intento de suicidio más recurrentes, es importante recordar que actualmente en el estado de Pernambuco -en donde se encuentra el Hospital da Restauração-, los niños accidentados son llevados de inmediato a las llamadas ‘Unidades de Pronto Atendimento' (UPA), de donde solo son transferidos a hospitales de referencia cuando la gravedad de sus lesiones lo amerita. Esto explica por qué no hubo una gran distribución para los tipos de accidentes: el Hospital da Restauração es referencia para el tratamiento de quemaduras.

Aunque no sea el mayor método de preferencia para los que tratan de terminar con su vida, las quemaduras también aparecen como uno de los medios utilizados para tal, recordando que son las mujeres las que más lo utilizan (Sá et al., 2010; Schnitman et al., 2010).

En relación al momento del accidente, en todos los casos hubo presencia de terceros, adultos o niños. De Lima, Barbisa Ximenes, Silva Joventino, Vieira, \& Oriá (2009), revelan que la presencia de un adulto no impide la ocurrencia de un accidente, una vez que muchos no saben cómo evitarlos, o están tan entrelazados a otras actividades que se vuelve incapaces de realizar una supervisión adecuada de los niños.

En algunos casos, las propias personas que estaban con los niños fueron agentes causantes de los accidentes. O sea, los niños se convirtieron en sujetos pasivos, existiendo contribución de otro para la ocurrencia del accidente. De esta manera, muchos pueden pensar que el accidente fue solo algo casual o azaroso, como pensaron los propios familiares cuando respondieron a las entrevistas al ser cuestionados sobre las motivaciones para que ocurrieran los incidentes -dijeron que habían sido, en gran parte, solamente accidentes.

De cualquier forma, se corrobora lo que Granel (2009) dijo, en cuanto a que si bien un accidente muestra ser un acto descoordinado para la lógica de la consciencia, en verdad se asemeja a un acto realizado por dos personas coordenadas entre sí, respondiendo a un propósito inconsciente que se transforma en el accidente. Esto es cierto, ya que la observación de esta investigación llevó, inclusive, a creer que muchos de los niños, antes del momento del accidente, estuvieron expuestos a múltiples factores de riesgo.

Además, acerca del conocimiento sobre los accidentes, el grupo de niños, aunque tuvo dudas y mezcló varios acontecimientos, demostró saber algo al respecto. Esto no significa que sea suficiente para que deseen o pretendan evitar los accidentes. Al final, todos los seres humanos son susceptibles a padecer de un accidente, y las respuestas para el 'porqué' o 'cómo' sucedieron los accidentes se transforman en una interrogativa hasta para los mismos adultos, que pueden ir a los fines obscuros del inconsciente, que responde al medio al cual el individuo corresponde, conectándose a su perfil psicológico y a sus rasgos de carácter.

En el grupo también hubo respuestas de los familiares respecto a cómo veían a los niños. Respondieron que los consideraban seres cariñosos, teniendo momentos de agresividad solamente cuando eran provocados/contrariados o en las relaciones entre hermanos. Así, hay que estar atento sobre la capacidad de soporte que tienen estos niños sobre sus disgustos y aburrimiento, ya que la agresividad y hostilidad se configuran en un síntoma de trastorno del control de los impulsos, en conjunto con la baja tolerancia a las frustraciones, llevándolos a ser incapaces de esperar por la satisfacción de los deseos (Correa, \& Perez Barrero, 2006).

Los comportamientos impulsivos y agresivos, con baja capacidad de autocontrol, son un común denominador entre la gran parte de los sujetos que comenten suicidio. Al final, la baja tolerancia a las frustraciones y la dificultad en sentir afectos negativos, así como la tendencia a acting-out, son características de las personas impulsivas que buscan resolver sus situaciones problemáticas de manera eficaz (Casullo, Bonaldi, \& Liporace, 2006; Correa, \& Perez Barrero, 2006).

En relación al dibujo hecho, los niños afirmaron haber dibujado a la familia real, lo que refleja que 
ellos estaban conectados al principio de realidad vivenciada. Se observó la valorización de las figuras paternas y fraternales. La desvalorización fue fuertemente representada, solamente, por las figuras fraternas, principalmente en omisión, que tiende a ser la desvalorización llevada al extremo.

Otro punto a considerar fue la gran omisión también de los propios niños al dibujarse a sí mismos, que aunque en niños menores podría considerarse normal, es llamativo en esta muestra, con niños entre los 6 y los 12 años. Esto refleja que estos niños podrían no estar aceptándose o sintiéndose rechazados por sus familias; esto también puede denotar un deseo de no pertenecer a esta unidad familiar, mostrando no tener influencia sobre sus miembros y carecer de afectos.

\section{Consideración final}

Hay que resaltar que algunos individuos pueden tener uno o más factores de riesgo y nunca llegar a la realización del acto suicida. Empero, hasta aquí, pudimos encontrar muchas características relacio-

\section{Referências}

Adorno, I. (1994). Conversando com crianças sobre a morte. Campinas, SP: Editorial Psy.

Amaral, L. R., \& Mattioli, O. C. (2003). Em busca dos significados dos acidentes infantis: um encontro com a casualidade, a negligência, a violência e a depressão. Revista de Psicologia da Unesp, 2(1), 59-70. Recuperado de http://186.217.160.122/revpsico/index.php/revista/article/view/15/29

Amorim, M. G. R., Medeiros, G. X., Benício, J. A., Oliveira, S. B., Santos, E. V., \& Sousa, F. F. (2012). Incidência e principais causas de acidentes domésticos em crianças na fase toddler e pré-escolar. Recuperado de http://coopex.fiponline.com.br/images/ arquivos/documentos/7.pdf

Barrero, S., Nicolato, R., \& Corrêa, H. (2011). Fatores de risco para o suicídio durante o ciclo da vida humana. Recuperado de http://www.medcenter.com/medscape $/$ content.aspx?id=22353\&langtype $=104$

Basile, H. S. (2005). El suicídio de los adolescentes en Argentina. Alcmeon 47, 12(3), 211-231. Recuperado de http://www.alcmeon.com.ar/12/47/01_Basile.htm

Bella, M., Fernández, R., \& Willington, J. M. (2010). Intento de suicidio en niños y adolescentes: de- nadas con una tendencia suicida. Se debe prestar atención a los niños que constantemente se deparan con conflictos familiares que podrían ser evitados o correctamente abordados entre los pequeños.

Es cierto que en el material del análisis de la investigación se encontró una gama de factores de riesgo del desarrollo infantil que puede sugerir que estos accidentes serían intentos enmascarados de suicidio; pero también es verdad que fueron encontrados factores protectores que deben destacarse para la prevención de los hechos auto-lesivos.

Ante todo esto, los adultos deben comprender que la madurez del ser humano implica no solamente crecimiento personal, sino también relacional, y que aún existe un vasto campo de estudio acerca de las manifestaciones autodestructivas en la infancia, pues sin lugar a dudas el tema no se acaba aquí. Los accidentes y suicidios son, en cualquier población, un grave problema de salud pública que exige nuestra constante atención, pues su prevención y control sigue siendo una tarea bastante ardua.

presión y trastorno de conducta disocial como patologías más frecuentes. Archivos Argentinos de Pediatría, 108(2), 124-129. Recuperado de http:// www.scielo.org.ar/scielo.php?script=sci_arttext\&pi$\mathrm{d}=$ S0325-00752010000200006

Bertolote, J. M., \& Fleischmann, A. (2002). A global perspective in the epidemiology of suicide. Suicidology, 7(2), 6-8. Recuperado de https://www.iasp.info/pdf/ papers/Bertolote.pdf

Cassorla, R. M. S. (Org.). (1991). Do suicídio: estudos brasileiros. São Paulo, SP: Papirus.

Cassorla, R. M. S. (2005). O que é suicídio. São Paulo, SP: Brasiliense.

Casullo, M. M., Bonaldi, P. D., \& Liporace, M. F. (2006). Comportamientos suicidas en la adolescencia: morir antes de la muerte. Buenos Aires: Lugar Editorial.

Corrêa, H., \& Perez Barrero, S. (2006). Suicídio: uma morte evitável. São Paulo, SP: Atheneu.

De Lima, R. P., Barbisa Ximenes, L., Silva Joventino, E., Vieira, L. J. E. S., \& Oriá, M. O. B. (2009). Acidentes na infância: local de ocorrência e condutas dos familiares no âmbito domiciliar. Enfermería Global, (15), 1-13. 
Delvan, J. S., Becker, A. P. S., \& Braun, K. (2010). Fatores de risco no desenvolvimento de crianças e a resiliência: um estudo teórico. Revista de Psicologia, 2(1), 349-357. 10.18256/2175-5027/psico-imed.v2n1p349-357

Dias, M. L. (1997). Suicídio: testemunhos de adeus. São Paulo, SP: Brasiliense.

Durkheim, E. (2009). El suicidio. Buenos Aires: Ediciones Libertador.

Fensterseifer, L., \& Werlang, B. (2003). Suicídio na infância: será a perda da inocência? Psicologia e Argumento, 21(35), 39-46. Recuperado de http://pesquisa.bvs.br/brasil/resource/pt/lil-437302

Franco, M. L. P. B. (2005). Análise de conteúdo (2a ed.). Brasília, DF: Liber Livros.

García, A. M., Muro, A. G., Prado, J. L. T., Martinez, N. D., \& Calvo, Y. N. (2007) Intento suicida en niños y adolescentes: aspectos epidemiológicos. Recuperado de http://www.bvs.sld.cu/revistas/gme/pub/ vol.9.\%282\%29_04/p4.html

Granel, J. A. (2009). Teoría psicoanalítica del accidentarse. Buenos Aires: Letra Viva.

Marchiori, H. (2006). El suicidio: enfoque criminológico. México: Editorial Porrúa.

Marin, A., \& Piccinini, C. A. (2009). Famílias uniparentais: a mãe solteira na literatura. Psico, 40(4), 422-429. Recuperado de http://revistaseletronicas.pucrs.br/ ojs/index.php/revistapsico/article/view/2683

Martins, C. B. G. (2006). Acidentes na infância e adolescência: uma revisão bibliográfica. Revista Brasileira de Enfermagem, 59(3), 344-348. doi:10.1590/S0034-71672006000300017

Meleiro, A. M. A. S., Teng, C. T., \& Wang, Y. P. (2004). Suicídio: estudos fundamentais. São Paulo, SP: Segmento Farma.

Nascimento, D. C., Sousa, M., \& Chacon, S. (2012). Acidentes domésticos na infância: a melhor forma de combatê-los é a prevenção. Juazeiro do Norte, Ceará: Universidade Federal do Ceará, Campus Cariri.

Oliveira, D., Siqueira, A. C., Dell'Aglio, D. D., \& Lopes, R. C. S. (2008). Impacto das configurações familiares no desenvolvimento de crianças e adolescentes: uma revisão da produção científica. Interação em Psicologia, 12(1), 87-98. doi:10.5380/psi.v12i1.9172

ONG Criança Segura (Brasil). (2011). Guia criança segura na escola. São Paulo, SP: o autor. Recuperado de http://ieij.com.br/CULTieij.2012/10/CULTieij.2012.10.Texto.GrupoDE.pdf
Paes, C. E. N., \& Gaspar, V. L. V. (2005). As injúrias não intencionais no ambiente domiciliar: a casa segura. Jornal de Pediatria (Rio J), 81(5), 146-154. doi:10.1590/S0021-75572005000700004

Pinheiro, M. \& Biasoli-Alves, Z. (2008). A família como base. In L. Weber, Família e desenvolvimento (pp. 21-36). Curitiba, PR: Juruá.

Polaino-Lorente, A., \& Heras, F. J. (1990). En torno al fracaso escolar como hipótesis justificatoria-explicativa del suicidio infantil. Revista Complutense de Educación, 1(2), 223-239. Recuperado de http:// revistas.ucm.es/index.php/RCED/article/view/ RCED9090230223A/18214

Ramires, V. R. R., Passarini, D. S., Flores, G. G., \& Santos, L. G. (2009). Fatores de risco e problemas de saúde mental de crianças. Arquivos Brasileiros de Psicologia, 61(2), 1-14. Recuperado de http://pepsic.bvsalud.org/pdf/arbp/v61n2/v61n2a12.pdf

Resmini, E. (2004). Tentativas de suicídio: um prisma para compreensão da adolescência. Rio de Janeiro, RJ: Revinter.

Sá, N. N. B., Oliveira, M. G. C., Mascarenhas, M. D. M., Yokota, R. T. C., Silva, M. M. A. A., \& Malta, D. C. (2010). Atendimentos de emergência por tentativas de suicídio, Brasil, 2007. Revista Médica de Minas Gerais, 20(2), 145-152.

Schnitman, G., Kitaoka, E. G., Arouca, G. S. S., Lira, A. L. S., Nogueira, D., \& Duarte, M. B. (2010). Taxa de mortalidade por suicídio e indicadores socioeconômicos nas capitais brasileiras. Revista Baiana de Saúde Pública, 34(1), 44-59. Recuperado de http:/ /inseer.ibict. $\mathrm{br} / \mathrm{rbsp} /$ index.php/rbsp/article/view/12

Stengel, E. (1987). Psicología del suicidio y los intentos suicidas. Buenos Aires: Horme.

Torres, W. C. (1999). A criança diante da morte. São Paulo, SP: Casa do Psicólogo.

Unglert, C. V. S., Siqueira, A. A. F., \& Carvalho, G. A. (1987). Características epidemiológicas dos acidentes na infância. Revista de Saúde Pública, 21(3), 234245. doi:10.1590/S0034-89101987000300008

Vendrusculo, T. M, Balieiro, C. R. B., Echevarría-Guanilo, M. E., Farina Junior, J. A., \& Rossi, L. A. (2010). Queimaduras em ambiente doméstico: características e circunstâncias do acidente. Revista Latino-Americana de Enfermagem, 1(3), 157-164. doi:10.1590/S0104-11692010000300021 
Wagner, A. (2002). Possibilidades e potencialidades da família: a construção de novos arranjos a partir do recasamento. In A. Wagner (Org.), Família em cena: tramas, dramas e transformaç̧̃es (pp. 23-38). Petrópolis, RJ: Vozes.

Wagner, A., \& Levandowski, D. C. (2008). Sentir-se bem em família: um desafio à diversidade. Textos \& Contextos, 7(1), 88-97. Recuperado de http://revistaseletronicas.pucrs.br/ojs/index.php/fass/article/view/3940

Winnicott, D. W. (2011). A família e o desenvolvimento individual. São Paulo, SP: Martins Fontes.

\section{Renata Paula Morais dos Santos}

Mestre pela Universidad de Ciencias Empresariales y Sociales, B.A., Argentina.

E-mail: renatapaula_morais@hotmail.com

\section{Mônica Cristina Batista de Melo}

Doutora pelo Instituto de Medicina Integral Prof. Fernando Figueira, Recife - PE. Brasil.

E-mail: monicacbmelo@gmail.com
Endereço para envio de correspondência: Rua Jorge de Lima, 245 - Alegro 1404. Imbiribeira. CEP: 51160-070. Recife - PE. Brasil

Recebido: 06/04/2016

Aprovado:21/07/2016

Received:04/06/2016

Approved:07/21/2016

Recibido: 06/04/2016

Aceptado: 21/07/2016

Cómo citar: Santos, R. P. M., \& Melo, M. C. B. (2016). Tendencia Suicida en Niños Accidentados. Psicologia: Ciência e Profissão, 36(3): 571-583. doi:10.1590/1982-3703001282016

Como citar: Santos, R. P. M., \& Melo, M. C. B. (2016). Tendência Suicida em Crianças Acidentadas. Psicologia: Ciência e Profissão, 36(3): 571-583. doi:10.1590/1982-3703001282016

How to cite: Santos, R. P. M., \& Melo, M. C. B. (2016). Suicidality in Hilly Children. Psicologia: Ciência e Profissão, 36(3): 571-583. doi:10.1590/1982-3703001282016 